Check for updates

Cite this: RSC Adv., 2018, 8, 10255

Received 7th January 2018

Accepted 8th March 2018

DOI: $10.1039 / c 8 r a 00180 d$

rsc.li/rsc-advances

\section{Photoelectrocatalytic application of palladium decorated zinc oxide-expanded graphite electrode for the removal of 4-nitrophenol: experimental and computational studies $\dagger$}

\author{
Eseoghene H. Umukoro, ${ }^{a}$ Moses G. Peleyeju, ${ }^{a}$ Azeez O. Idris, ${ }^{a}$ Jane C. Ngila, ${ }^{\text {ab }}$ \\ Nonhlangabezo Mabuba, ab Lydia Rhyman, ac Ponnadurai Ramasami*ac \\ and Omotayo A. Arotiba iD *ab
}

\begin{abstract}
A novel $\mathrm{Pd}-\mathrm{ZnO}$-expanded graphite (EG) photoelectrode was constructed from a $\mathrm{Pd}-\mathrm{ZnO}$-EG nanocomposite synthesised by a hydrothermal method and characterised using various techniques such as X-ray diffractometry (XRD), Raman spectroscopy, UV-Vis diffuse reflectance spectroscopy, nitrogen adsorption-desorption analysis, transmission electron microscopy (TEM), scanning electron microscopy (SEM) and energy dispersive spectrometry (EDS). Cyclic voltammetry and photocurrent response measurements were also carried out on the electrode. The $\mathrm{Pd}-\mathrm{ZnO}-\mathrm{EG}$ electrode was employed in the photoelectrocatalytic removal of 4-nitrophenol as a target water pollutant at a neutral $\mathrm{pH}$ and with a current density of $7 \mathrm{~mA} \mathrm{~cm}{ }^{-2}$. Optical studies revealed that the $\mathrm{Pd}-\mathrm{ZnO}-\mathrm{EG}$ absorbed strongly in the visible light region. The $\mathrm{Pd}-\mathrm{ZnO}-\mathrm{EG}$ electrode showed improved photoelectrocatalytic activity in relation to $\mathrm{ZnO}-\mathrm{EG}$ and $\mathrm{EG}$ electrodes for the removal of the 4-nitrophenol. The photocurrent responses showed that the $\mathrm{Pd}-\mathrm{ZnO}-\mathrm{EG}$ nanocomposite electrode could be employed as a good photoelectrode for photoelectrocatalytic processes and environmental remediation such as treatment of industrial waste waters. Density functional theory method was used to model the oxidative degradation of 4-nitrophenol by the hydroxyl radical which generates hydroquinone, benzoquinone, 4-nitrocatechol, 4-nitroresorcinol and the opening of the 4-nitrophenol ring. Furthermore, the hydroxyl radical is regenerated and can further oxidise the ring structure and initiate a new degradation process.
\end{abstract}

\section{Introduction}

The efficient and effective disposal of organic pollutants in the environment is a necessity for sustainable development of society. However, this is a major challenge due to the growth of industrialisation. ${ }^{1}$ Nitrophenol and its derivatives are organic pollutants which are mostly used in agricultural, textile, paper, explosives and pharmaceutical industries., ${ }^{2,3}$ They are potentially hazardous and mutagenic to humans and the general ecosystem., ${ }^{2,4}$ Among the derivatives of nitrophenols, 4-nitrophenol is found to be highly toxic, stable and biorefactory in nature. ${ }^{2,4}$ Therefore, the removal of 4-nitrophenol from industrial wastewaters is essential. Several traditional methods have been utilised for the treatment of these 4-nitrophenol-containing

${ }^{a}$ Department of Applied Chemistry, University of Johannesburg, South Africa. E-mail: oarotiba@uj.ac.za; p.ramasami@uom.ac.mu

${ }^{b}$ Centre for Nanomaterials Science Research, University of Johannesburg, South Africa

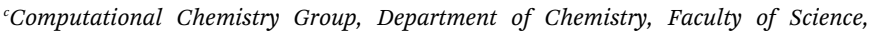
University of Mauritius, Réduit 80837, Mauritius

$\dagger$ Electronic supplementary information (ESI) available. See DOI: $10.1039 / \mathrm{c} 8 \mathrm{ra} 00180 \mathrm{~d}$ wastewaters, but the poor removal rate is still a general concern due to its high resistance to biodegradation..$^{5-8}$

In recent years, electrochemical methods have been successfully developed as alternatives to the conventional methods for the removal of organic pollutants. This is mainly due to the generation of highly reactive hydroxyl radical for the non-selective oxidation of these pollutants. ${ }^{9-11}$ This process entails the electrochemical production of hydroxyl radical at the surface of the electrode which is followed by its reaction with the organic pollutants., ${ }^{\mathbf{9 1 1}}$ The advantages of electrocatalytic degradation technique include the immobilisation of the catalyst (electrode) which helps in minimising catalyst separation from the reaction system, controlled reaction conditions and the relatively low equipment cost. ${ }^{\mathbf{1 0 , 1 2}}$ However, some drawbacks have also been reported such as the oxygen evolution which takes place simultaneously with the oxidation of the pollutant resulting from the application of high voltage, the low concentration of the pollutant raises the problem of mass transfer and the processes can be time consuming..$^{\mathbf{1 0 , 1 3 , 1 4}}$ Thus, it is important to improve this technique by the concurrent use of visible light irradiation (photolysis), leading to a process known as 
photoelectrocatalysis. In this process, a photoanode is constructed by mounting a semiconductor material that is photoactive on a conducting support with the simultaneous application of light and a bias voltage. This leads to the production of non-selective oxidants such as hydroxyl radical by the semiconductor and the conducting support for the degradation of the pollutants. ${ }^{\mathbf{9}, 15} \mathrm{TiO}_{2}$ semiconductor photoanode is the most widely used semiconductor photoanode. ${ }^{\mathbf{1 4 - 2 0}}$ Nevertheless, in recent years, $\mathrm{ZnO}$ is being utilised as an alternative to $\mathrm{TiO}_{2}$ due to the nontoxicity, availability, low cost, chemical and thermal stability, good catalytic efficiency and optoelectronic nature of $\mathrm{ZnO}^{21-24} \mathrm{ZnO}$ has also found applications in photovoltaic devices, water splitting and organic pollutants degradation. ${ }^{25,26}$ However, the fundamental problem of $\mathrm{ZnO}$ is the recombination rate of the photoexcited holes and electrons which hinders its photocatalytic efficiency. Generally, the kinetics of the recombination rate is faster than that of the surface reduction-oxidation reactions, and this results in a decrease of the quantum efficiency of photocatalysis. ${ }^{23,24}$ Different techniques have been used to reduce the electronhole recombination rate and these include the introduction of semiconductor oxides, carbonaceous materials and noble metals. ${ }^{23,24,27}$ Noble metals nanoparticles such as Au, Ag, Pt and Pd have been reported to enhance the photocatalytic activity of $\mathrm{ZnO} .^{28-30}$ In comparison with $\mathrm{Pt}$ and $\mathrm{Au}, \mathrm{Pd}$ is cheaper and it has been employed in catalytic processes. ${ }^{24} \mathrm{Pd}$ nanoparticles can act as collectors for the photoexcited electrons and these aid the charge transfer and transportation processes, thereby creating more active sites for the catalytic reaction. ${ }^{31}$ The use of expanded graphite (EG) can also help in reducing the recombination rate of $\mathrm{ZnO}$. EG is a carbonaceous material consisting of two dimensional bonded carbons which possesses excellent electrical conductivity, large surface area, good porosity, compressibility, low density and excellent electron transfer and transport properties. In addition, it possesses good mechanical and thermal stability, good resistance to high temperature, and can withstand high voltage. ${ }^{152-34}$ The inclusion of EG in PdZnO-EG can result in the EG acting as an electron sink for photoexcited electrons from $\mathrm{ZnO}$ due to the unique electron transport capability of EG. ${ }^{19}$ As a result, the problem of electron-hole recombination rate of $\mathrm{ZnO}$ would be minimised. Thus, the photoactivity of $\mathrm{ZnO}$ in the Pd-ZnO-EG nanocomposite would be enhanced by forming more oxidants such as hydroxyl radical for the degradation of organic pollutants. The EG can also be used as a conducting support for the immobilisation and trapping of the $\mathrm{ZnO}$ and $\mathrm{Pd}$, therefore, minimising the losses from the recovery of these photocatalysts. $^{15}$

Considering the unique properties of $\mathrm{Pd}, \mathrm{ZnO}$ and EG, it can be hypothesised that Pd-ZnO-EG nanocomposite can act as a promising photoeletrocatalytic material for the degradation of 4-nitrophenol. In this novel study, Pd-ZnO supported on EG was synthesised, fabricated into a photoanode and used for the photoelectrocatalytic removal of 4-nitrophenol as a model organic pollutant. Of importance is the understanding of the degradation pattern and products during the photoelectrocatalytic process because it can be used to estimate the toxicity of the product in comparison with the initial organic pollutants, the time of reaction, the kinetics and mechanism of reaction. To better understand the degradation mechanism, we have employed density functional theory (DFT) method to predict the degradation products and the different possible degradation pathways for the 4-nitrophenol degradation which is in agreement with experimental findings reported in literature.

\section{Experimental section}

\subsection{Chemicals}

Nitric acid, sulphuric acid, natural graphite, sodium sulphate, zinc acetate dehydrate, potassium hexacyanoferrate(II), sodium hydroxide, potassium chloride, 4-nitrophenol and potassium hexacyanoferrate(III). These were bought from Sigma Aldrich, Germany and used for the experiment without further purification.

\subsection{Synthesis of expanded graphite (EG)}

The expanded graphite was prepared by employing our previous technique. ${ }^{35,36}$ Sifted flakes of natural graphite $(300 \mu \mathrm{m})$ were immersed in a $1: 3(\mathrm{vol} / \mathrm{vol})$ mixture of concentrated nitric and sulphuric acids for $48 \mathrm{~h}$ at ambient conditions for the intercalation of the graphite material. The intercalated graphitic material was washed properly to achieve a $\mathrm{pH}$ value close to 7 , and dried in the air. Then, the expansion of the graphitic material was done by heating the material at about $800{ }^{\circ} \mathrm{C}$ to form a puffed-up material called expanded graphite.

\subsection{Synthesis and construction of Pd-ZnO-EG nanocomposite}

Zinc acetate dehydrate (3.29 g), $2.4 \mathrm{~g}$ of sodium hydroxide and $2 \mathrm{~g}$ of the prepared EG were mixed in $70 \mathrm{~mL}$ of ethanol solution and stirred for $30 \mathrm{~min}$. The mixture was poured into a Teflonlined autoclave and thermally treated at $160{ }^{\circ} \mathrm{C}$ for $24 \mathrm{~h}$. The autoclave was left to cool to ambient temperature. The ZnO-EG formed was centrifuged, washed several times with water and ethanol, and dried overnight at $60{ }^{\circ} \mathrm{C}$ in an oven. In order to prepare the Pd-ZnO-EG, a certain amount of the ZnO-EG was weighed and dispersed in ethanol, followed by ultrasonication for $30 \mathrm{~min}$. Palladium(II) acetate in ethanol solution was added and ultrasonicated further for $20 \mathrm{~min}$. The resulting mixture was put in a water bath and kept at $70{ }^{\circ} \mathrm{C}$ for $3 \mathrm{~h}$. The mixture was filtered and dried at $60{ }^{\circ} \mathrm{C}$ overnight. The amount of $\mathrm{Pd}$ in the composite was $5 \%$ by weight of the Pd-ZnO-EG nanocomposite.

\subsection{Construction of Pd-ZnO-EG, ZnO-EG and EG electrodes}

The construction of the electrodes was done using a technique described in our previous studies..$^{\mathbf{1 1}, 15,19,37}$ The as-prepared EG, ZnO-EG and Pd-ZnO-EG nanocomposites were used to make pellets using a hydraulic press at a pressure of about $10000 \mathrm{psi}$. These pellets were utilised for fabricating the electrodes. Copper wire, conductive silver paint and epoxy sealer were also used. These pellets were placed on the coiled copper wire with 
the assistance of the conductive silver paint. This was left out in ambient air overnight. The pellets edges were then sealed with the epoxy sealer in order for electricity to flow only from the basal plane. This was placed in a glass rod before further use.

\subsection{Characterisation of EG, ZnO-EG and Pd-ZnO-EG nanocomposites}

X-ray diffractometer (Philips PAN Analytical X'Pert powder, Netherlands) with $\mathrm{Cu}-\mathrm{K} \alpha$ radiation $(\lambda=0.15418)$ was used to determine the crystallinity of the as-prepared materials. The Raman spectra were measured with Witec alpha300 $\mathrm{R}$ confocal Raman microscope (Germany). The spectra from the UV absorbance were done at a wavelength of 200-800 $\mathrm{nm}$ with Shimadzu UV-2450 (Japan). Morphological images of the asprepared samples were taken with scanning electron microscopy (TESCAN, VEGA 3 XMU, Czech Republic) and transmission electron microscopy (JEOL JEM-2100, USA) at $200 \mathrm{kV}$ accelerating voltage. Energy-dispersive X-ray spectrometer (TESCAN, Czech Republic) connected to the SEM was employed in measuring the samples' elemental composition. The extent of mineralisation was measured with Teledyne Tekmar TOC Analyzer (USA). Cary 60 UV-Visible spectrophotometer (Agilent technologies, USA) was utilised in determining the removal efficiency of the 4-nitrophenol. The source of visible light irradiation was Oriel LCA-100 Solar Simulator (USA) which was equipped with $100 \mathrm{~W}$ xenon lamp and AM1.5G filter.

\subsection{Photoelectrochemical measurements}

Electrochemical and photoelectrochemical behaviours of the EG, ZnO-EG and Pd-ZnO-EG nanocomposite electrodes were investigated in a redox probe of $5 \mathrm{mM}$ of potassium ferrocyanide and ferricyanide in $0.1 \mathrm{M} \mathrm{KCl}$ solution. Cyclic voltammetry (CV) and chronoamperometry photocurrent responses under visible light (Oriel LCA-100 Solar Simulator) were investigated in a three-electrode electrochemical configuration with Autolab $302 \mathrm{~N}$ potentiostat (The Netherlands), while the working electrodes (with diameter of $1.3 \mathrm{~cm}$ and geometric area of $1.33 \mathrm{~cm}^{2}$ ) were the as-prepared materials, platinum foil was used as counter electrode and the reference electrode was $\mathrm{Ag} / \mathrm{AgCl}$ (3.0 M KCl).

\subsection{Electrochemical and photoelectrocatalytic experiments}

The photoelectrocatalytic activity of the materials were determined by the degradation of 4-nitrophenol. This was carried out in a reactor $(100 \mathrm{~mL})$ with $20 \mathrm{ppm}$ of 4-nitrophenol. A solution of $0.1 \mathrm{M} \mathrm{Na}_{2} \mathrm{SO}_{4}$ was used as supporting electrolyte. The electrodes were positioned to face the irradiation from the solar simulator. The source of power was the potentiostat, while Oriel LCA-100 Solar Simulator was the source of irradiation. In order to achieve a power beam of 1 sun which is equivalent to a 100 $\mathrm{mW} \mathrm{cm}^{-2}$, an AM1.5G filter was placed in the simulator and the reactor was $8 \mathrm{~cm}$ away from the irradiation source. A bias potential was applied without making use of the visible light for the electrocatalytic removal of the 4-nitrophenol. At $25 \mathrm{~min}$ intervals, an aliquot of 4-nitrophenol was withdrawn from the reactor with disposable syringe over a period of $2 \mathrm{~h} 30 \mathrm{~min}$. The aliquot was then filtered and the removal efficiency of the 4nitrophenol was measured on a UV-Visible spectrophotometer. The total organic content was investigated on a TOC analyser (Teledyne Tekmar TOC fusion). Furthermore, the influence of $\mathrm{pH}$, current density and percentage of $\mathrm{Pd}$ in the nanocomposite were studied.

\subsection{Computational details}

Quantum chemical calculations based on density functional theory (DFT) method were used to study the different possible pathways for the degradation of 4-nitrophenol. Full geometry optimisations were carried out in the gas phase with the B3LYP $^{38,39}$ functional with the 6-31G(d) basis set. ${ }^{40}$ The optimisations were followed by frequency computations at the same level of theory to confirm that the stationary points are minima (no imaginary frequencies) and that each of the transition state (TS) structures has only one imaginary frequency. The reaction pathways were subjected to intrinsic reaction coordinate (IRC) ${ }^{41,42}$ analysis in order to trace their paths and to confirm that the optimised TS connects the correct reactant and product. Zero-point energy (ZPE) correction is included in all the reported electronic energies. All the computations were carried out using the Gaussian 09 software suite ${ }^{43}$ running on the GridChem Science Gateway. ${ }^{44-46}$

\section{Results and discussion}

\subsection{Raman studies}

The Raman spectra of the EG and Pd-ZnO-EG materials are illustrated in Fig. 1a and b. The band observed at $100 \mathrm{~cm}^{-1}$ of the Raman spectrum of $\mathrm{ZnO}$ (Fig. 1a) corresponds to the $\mathrm{E}_{2}$ (Low) mode while the peak at $336 \mathrm{~cm}^{-1}$ results from the Raman active frequency phonons, $\mathrm{E}_{2}$ (High) $-\mathrm{E}_{2}$ (Low). The peak around $388 \mathrm{~cm}^{-1}$ can be assigned to the $\mathrm{A}_{1 \mathrm{~T}}$ mode, while the low intensity peak at $588 \mathrm{~cm}^{-1}$ can be ascribed to the $\mathrm{E}_{1 \mathrm{~L}}$ mode. In addition, the very intense and characteristic peak at $441 \mathrm{~cm}^{-1}$ is attributed to $\mathrm{E}_{2}$ (high), considered as the Raman active optical phonon mode. This is an indication that the as-prepared $\mathrm{ZnO}$ possesses a wurtzite hexagonal phase. ${ }^{47-49}$ The peak at $639 \mathrm{~cm}^{-1}$ is exhibited by Pd nanoparticles which is a Raman active vibrational mode and characteristic feature of PdO..50,51 In Fig. 1b, it is clear that additional new peaks appeared near 1353 and $1587 \mathrm{~cm}^{-1}$ which are the D and $\mathrm{G}$ bands of the EG, respectively (inset). These are due to the in-plane phonon vibration of the $\mathrm{sp}^{2}$-bonded carbon atoms of the graphite material ( $G$ band) and the defects produced in the graphite sheets ( $\mathrm{D}$ band) due to the formation of $\mathrm{sp}^{3}$ carbon bonding caused by the oxygen groups present in the EG. However, a disappearance of the two peaks at 388 and $588 \mathrm{~cm}^{-1}$ was observed. In addition, shifts were noticed in the peaks at 330 and $439 \mathrm{~cm}^{-1}$ and this could be due to the incorporation of the Pd and EG. ${ }^{52,53}$ This indicates the successful preparation of the Pd-ZnO-EG nanocomposite. 
(a)

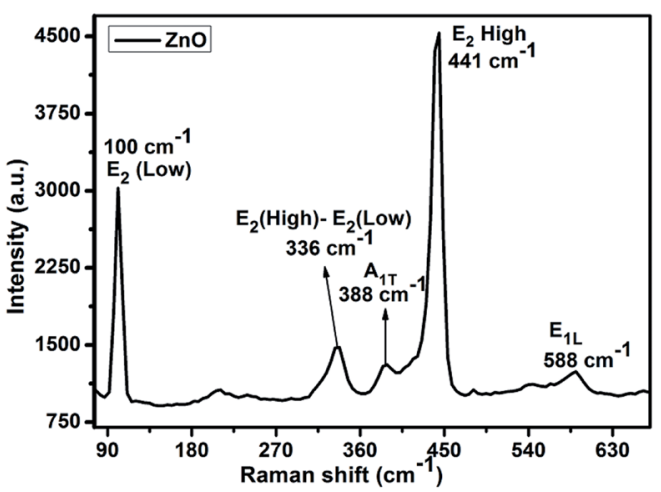

(b)

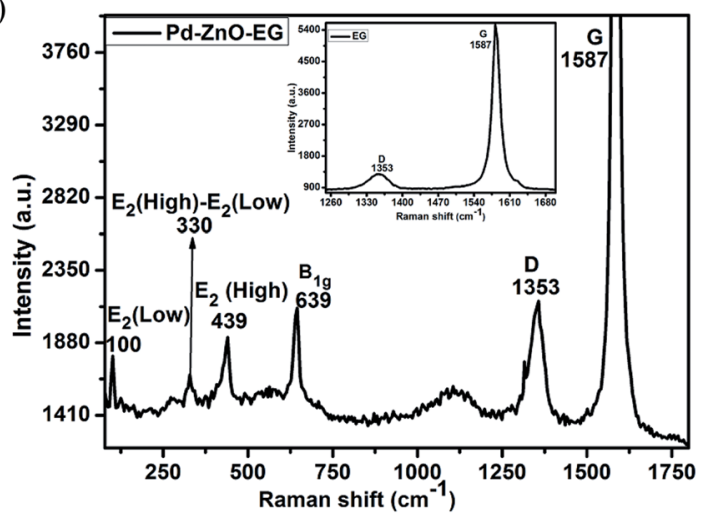

(c)

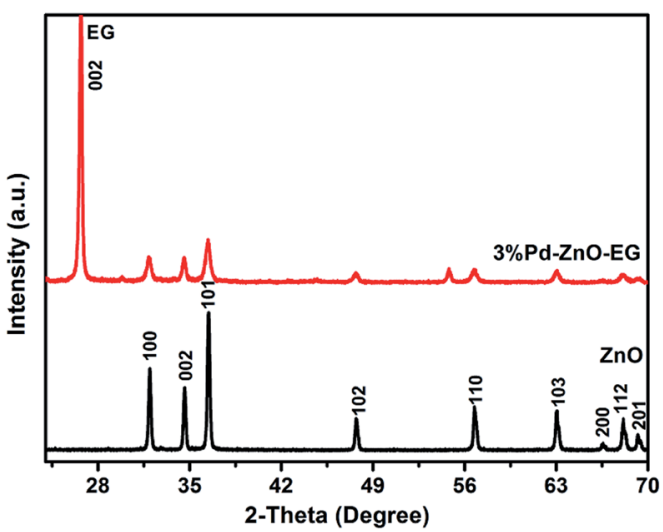

(d)

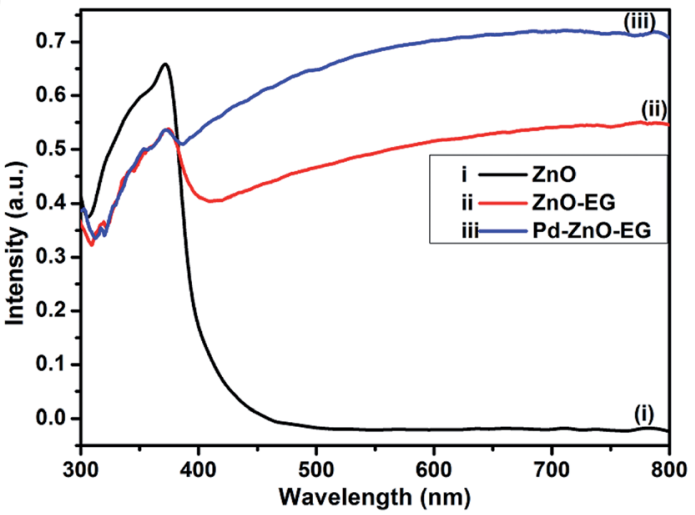

Fig. 1 Raman spectra of (a) ZnO and (b) Pd-ZnO-EG and EG (inset); (c) $X$-ray diffractograms of $\mathrm{ZnO}$ and $3 \% \mathrm{Pd}-\mathrm{ZnO}-\mathrm{EG}$; (d) UV-Visible absorbance spectra of (i) $\mathrm{ZnO}$, (ii) $\mathrm{ZnO}-\mathrm{EG}$ and (iii) $\mathrm{Pd}-\mathrm{ZnO}-\mathrm{EG}$.

\subsection{XRD analysis}

The crystallinity and phase of the samples were examined by conducting XRD analysis. The XRD patterns of $\mathrm{ZnO}$ and $\mathrm{Pd}-$ ZnO-EG are presented in Fig. 1c. The XRD pattern of ZnO shows peaks at $2 \theta=31.8^{\circ}, 34.6^{\circ}, 36.4^{\circ}, 47.7^{\circ}, 56.7^{\circ}, 63.0^{\circ}, 66.5^{\circ}$ and $68.1^{\circ}$ which correspond to the crystal plane of (100), (002), (101), (102), (110), (103), (200) and (112). These confirm that the hexagonal wurtzite structure of $\mathrm{ZnO}$ was prepared (JCPDS Card no. 36-1451). The conspicuous peak observed at $2 \theta=26.46^{\circ}$ is allocated to the (002) crystal plane of EG, confirming the presence of EG. However, due to the small amount of Pd added, the peaks for Pd were not observed. ${ }^{24}$

\subsection{UV-visible analysis}

The optical behaviour of the materials was determined using UV-Visible analysis. The ZnO-EG exhibited a better photoabsorbance than the $\mathrm{ZnO}$ in the region of visible light from $\lambda$ $=430-800 \mathrm{~nm}$ (Fig. 1d). This can be attributed to the presence of EG which is photosensitizing in nature. ${ }^{54}$ It can also be observed that Pd-ZnO-EG displayed a better photo-response in the visible light region with a higher intensity relative to the asprepared ZnO-EG. Firstly, this better photoresponse is an indication of the addition of Pd to the ZnO-EG material. Secondly, the decoration of the ZnO-EG with Pd aided in effective separation and transport of the photogenerated charge carriers. ${ }^{55}$ In the same vein, the surface plasmon resonance behaviour of $\mathrm{Pd}$ played a role in improving the light absorption. ${ }^{56}$ It can be deduced that the photosensitizing nature and charge transfer ability of the EG as well as the surface plasmon resonance behaviour of the Pd resulted in a synergic enhancement of the photoactivity observed in the Pd-ZnO-EG nanocomposite. ${ }^{15,55}$

\subsection{Brunauer-Emmett-Teller (BET) analysis}

The determination of the surface area, pore volume and pore size of the ZnO and Pd-ZnO-EG was carried out with the aid of nitrogen adsorption-desorption (BET) analysis. Table 1 shows the obtained results from the analysis. The nitrogen adsorption-desorption isotherms of the prepared Pd-ZnO-EG sample are presented (ESI Fig $\mathrm{S} 1 \dagger$ ). It was observed that the Pd-ZnO-EG displayed bigger pore size, pore volume and BET surface area in comparison with the bare $\mathrm{ZnO}$. This is an indication that the incorporation of the Pd and EG led to an increase in surface area which can improve the photoelectrocatalytic degradation of the 4-nitrophenol by the Pd-ZnO-EG electrode.

Table 1 BET surface area, pore size and pore volume of the bare $\mathrm{ZnO}$ and $\mathrm{Pd}-\mathrm{ZnO}$-EG samples

\begin{tabular}{llll}
\hline Material & $\begin{array}{l}\text { BET surface } \\
\text { area }\left(\mathrm{m}^{2} \mathrm{~g}^{-1}\right)\end{array}$ & $\begin{array}{l}\text { Pore volume } \\
\left(\mathrm{cm}^{3} \mathrm{~g}^{-1}\right)\end{array}$ & $\begin{array}{l}\text { Pore size } \\
(\mathrm{nm})\end{array}$ \\
\hline ZnO & 3.2959 & 0.0105 & 12.7215 \\
Pd-ZnO-EG & 4.9329 & 0.0354 & 28.6602
\end{tabular}




\subsection{Morphological studies}

The morphological images of the as-prepared ZnO, EG, and PdZnO-EG were taken on SEM and TEM, while the elemental composition were determined using an energy dispersive X-ray spectroscopy joined to the TEM. Fig. 2a shows the different layers of graphitic sheets and pores of the EG for the immobilisation of the Pd and $\mathrm{ZnO}$ nanoparticles. In Fig. 2b, the $\mathrm{ZnO}$ nanoparticles are supported within the layers of the EG sheets. Fig. 2c illustrates the TEM image of the ZnO particles. The scale shows that most of the particles are in the nano regime. Fig. $2 \mathrm{~d}$ depicts the TEM image of Pd-ZnO-EG where all the three components can be seen. The Pd nanoparticles (smaller nanoparticles) are distributed on the $\mathrm{ZnO}$ nanoparticles (larger particles) within the graphitic sheets/layers of the EG. In Fig. 2e, the EDS spectrum shows the elemental composition of the Pd$\mathrm{ZnO}-\mathrm{EG}$ to be $\mathrm{C}, \mathrm{O}, \mathrm{Zn}$ and $\mathrm{Pd}$, confirming the successful preparation of the Pd-ZnO-EG nanocomposite. ${ }^{24}$

\subsection{Photoelectrochemical characterisation}

The cyclic voltammetry of the Pd-ZnO-EG displayed a much higher current in relation to EG and ZnO-EG electrodes (Fig. 3a). Lower faradaic currents of the $\left[\mathrm{Fe}(\mathrm{CN})_{6}\right]^{-3 /-4}$ were observed from the EG and ZnO-EG when compared to that generated from Pd-ZnO-EG. This may be due to the catalytic property of the Pd nanoparticles which were added to the ZnO-EG to produce the Pd-ZnO-EG material. ${ }^{24}$ The inclusion of $\mathrm{Pd}$ increased the electroactive surface area of the electrode, thus the Pd-ZnO-EG is seen to exhibit a higher rate of electrode reaction. ${ }^{24,35,55}$ To support this argument, the electroactive surface areas of the electrodes were calculated using the Randles-Sevcik equation;

$$
i_{\mathrm{p}}=k n^{3 / 2} A D^{1 / 2} v^{1 / 2} C
$$

where $D$, diffusion co-efficient, is $7.6 \times 10^{-6}$ for ferrocyanide, $k$ is 2.69 is $10^{5}, A$ is the area of the electrode active surface, $n$ is the number of electrons involved in the reaction process, $C$ is concentration of the electrochemical probe and $v$ is the scan rate. ${ }^{57,58}$ The calculated electroactive surface areas of the EG, ZnO-EG and Pd-ZnO-EG electrodes are 1.60, 2.27 and 10.91 $\mathrm{mm}^{2}$ respectively. These suggest that there was a considerable increase in the electrochemical active sites after the addition of the Pd nanoparticle. ${ }^{35}$ Chronoamperometry technique was employed to study the photocurrent responses of the EG, ZnOEG and Pd-ZnO-EG materials. The efficiency of charge carriers separation and transfer is proportional to the photocurrent response of the material. ${ }^{17,59,60}$ It is observed from Fig. $3 \mathrm{~b}$ that the Pd-ZnO-EG exhibited a stronger photoresponse in comparison to the ZnO-EG and EG materials. This suggests that the Pd nanoparticles could effectively retard the recombination of the photo-generated electrons and holes, and thereby improving the photoelectrocatalytic activity of the Pd-ZnO-EG, thus, making it a promising photoelectrode for photoelectrocatalytic processes especially in water treatment process. (a)

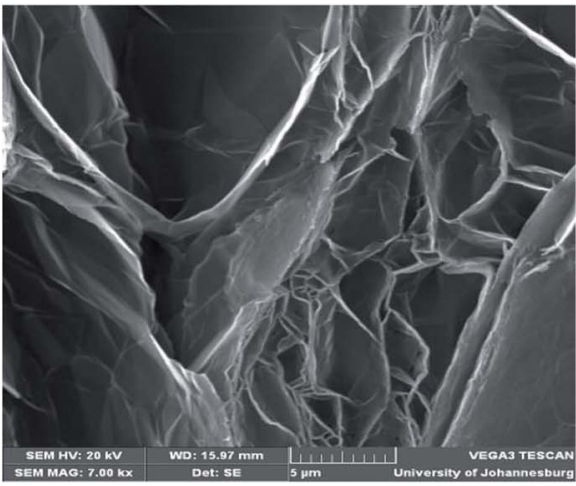

(b)

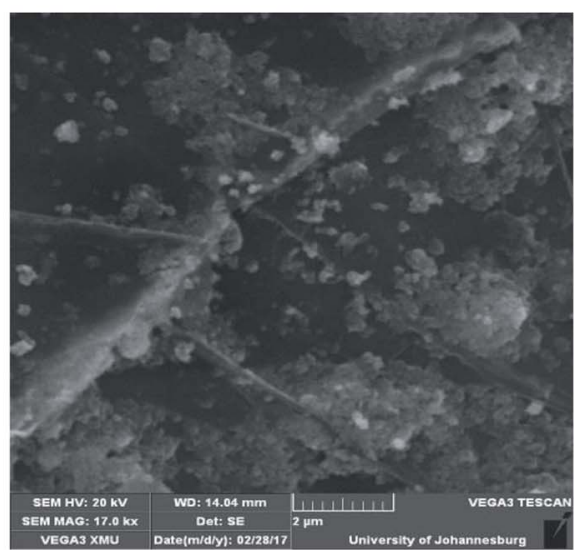

(c)

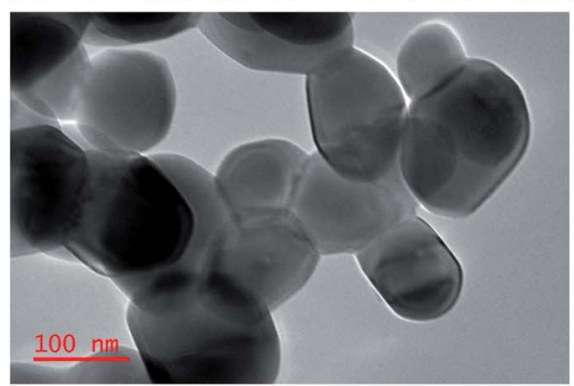

(d)

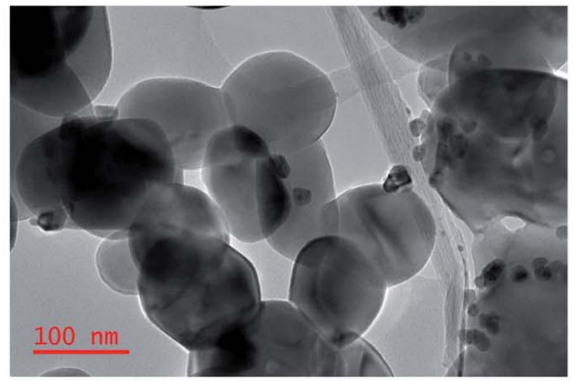

(e)

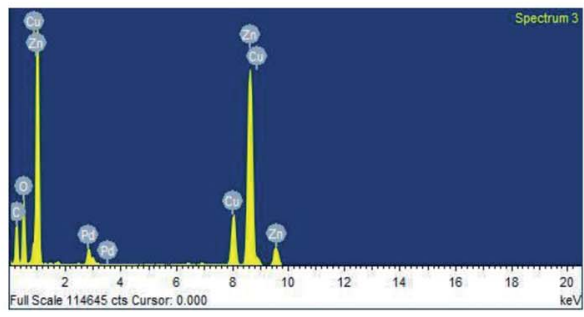

Fig. 2 SEM images of (a) expanded graphite, (b) $\mathrm{Pd}-\mathrm{ZnO}$ nanoparticles trapped in EG sheets. TEM images of (c) $\mathrm{ZnO}$ and (d) $\mathrm{Pd}$ nanoparticles anchored on $\mathrm{ZnO}$ immobilised on the EG. (e) EDX spectra showing the presence of $\mathrm{Pd}, \mathrm{Zn}, \mathrm{O}$ and $\mathrm{C}$ in the $\mathrm{Pd}-\mathrm{ZnO}-\mathrm{EG}$ samples. 

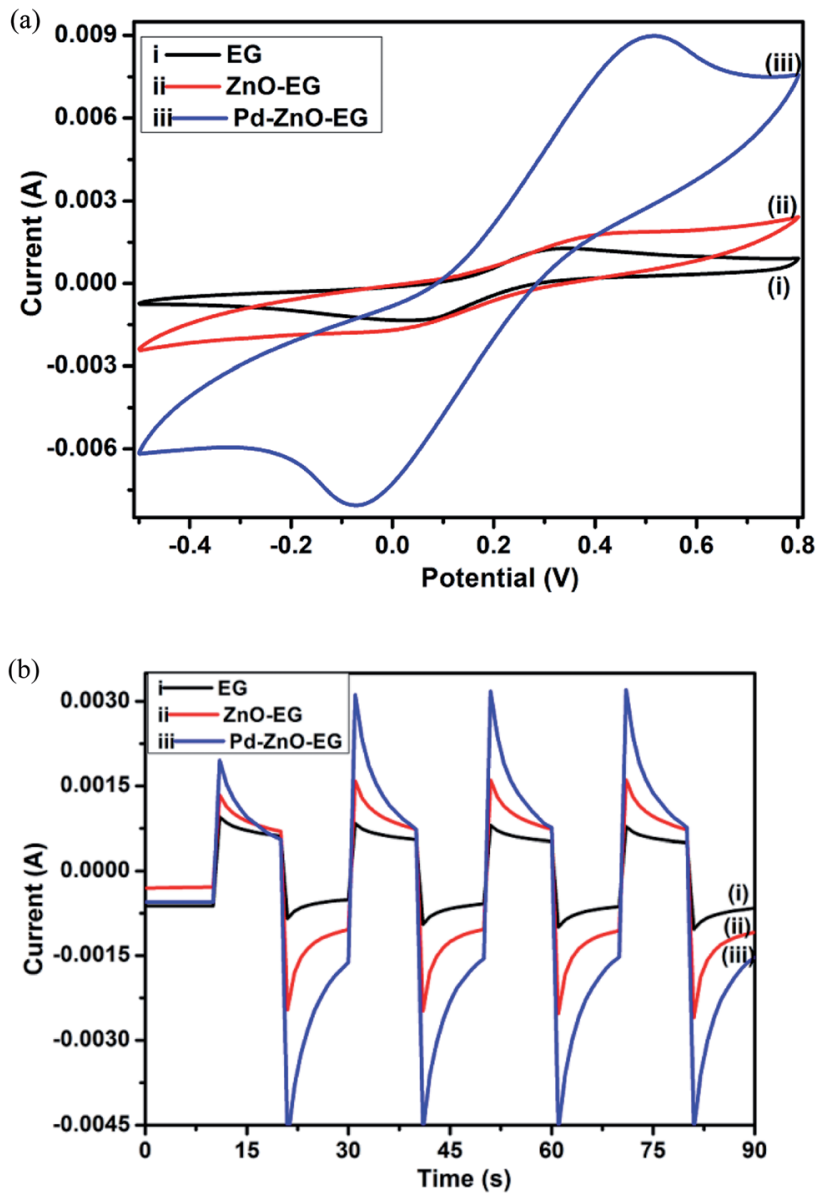

Fig. 3 (a) Cyclic voltammograms of (i) EG, (ii) $\mathrm{ZnO}-\mathrm{EG}$ and (iii) $\mathrm{Pd}-$ $\mathrm{ZnO}-\mathrm{EG}$ electrodes in $5 \mathrm{mM}\left[\mathrm{Fe}(\mathrm{CN})_{6}\right]^{-3 /-4}, 0.1 \mathrm{M} \mathrm{KCl}$ solution, at $50 \mathrm{mV} \mathrm{s}^{-1}$ scan rate (b) photocurrent responses of (i) $E G$, (ii) $\mathrm{ZnO}-\mathrm{EG}$ and (iii) $\mathrm{Pd}-\mathrm{ZnO}-\mathrm{EG}$ electrodes in the dark and under light.

\subsection{Photoelectrocatalytic experiments}

The photoelectrocatalytic activities of the EG, ZnO-EG and PdZnO-EG electrodes were evaluated by the rate of removal of 4nitrophenol as a target organic pollutant. The removal rate was monitored using UV-Visible spectroscopy and total organic carbon (TOC) analysis. For control experiments, photolytic, photocatalytic and electrocatalytic removal of 4-nitrophenol were carried out and compared with the photoelectrocatalytic process using the Pd-ZnO-EG material. ${ }^{61}$ The results revealed that the removal of 4 -nitrophenol was $20 \%$ for photolysis, $31 \%$ for photocatalysis, $69 \%$ for electrocatalysis and $94 \%$ for the photoelectrocatalytic process (Fig. 4a). Furthermore, the EG and ZnO-EG electrodes were used as control experiments for the photoelectrocatalytic removal of 4-nitrophenol. As presented in Fig. $4 \mathrm{~b}$, the kinetics curves show that the $\mathrm{ZnO}$-EG electrode gave a better removal efficiency compared to that of the EG electrode. This is attributed to the photoactive nature of $\mathrm{ZnO}$ in the $\mathrm{ZnO}$ EG material. Moreover, after the addition of Pd to the ZnO-EG material, an enhanced photoelectrocatalytic removal performance (94\%) was found. The improved removal efficiency is as a result of the photoactive nature of $\mathrm{ZnO}$ and the enhanced (a)

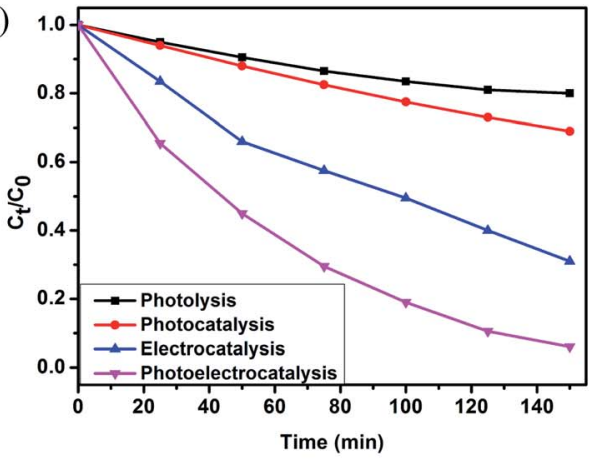

(b)

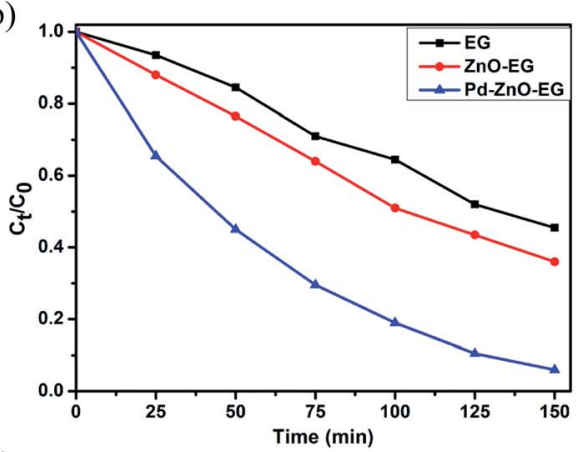

(c)

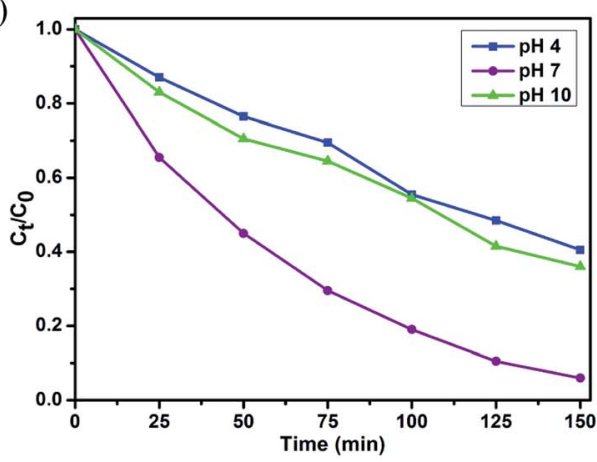

(d)

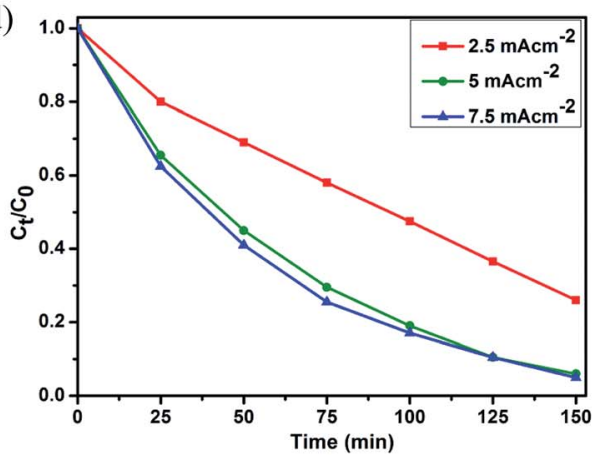

Fig. 4 Kinetics curves of (a) electrochemical and photoelectrocatalytic removal of 4-nitrophenol at Pd-ZnO-EG electrode. (b) Photoelectrocatalytic removal of 4-nitrophenol at EG, ZnO-EG and $\mathrm{Pd}-\mathrm{ZnO}-\mathrm{EG}$ electrodes. Effect of various (c) $\mathrm{pH}$ conditions and (d) different current densities at $\mathrm{Pd}-\mathrm{ZnO}-\mathrm{EG}$ electrode.

absorption of visible light caused by the surface plasma resonance ability of Pd in the composite. The Pd can also behave as electron sink for the photoinduced electrons, thereby minimising the recombination of the electron-hole pairs generated. ${ }^{\mathbf{2 4 , 6 2}}$ In addition, the ability of the EG to act as electron sink 
as well as its electron transport nature must have aided in inhibiting the recombination of the photoinduced holes and electrons which led to the improved photoelectrocatalytic performance of the Pd-ZnO-EG electrode. The synergic combination of $\mathrm{ZnO}$, EG and Pd that led to improved photocatalytic degradation can be buttressed with photocurrent responses in Fig. 3b which was highest for the Pd-ZnO-EG electrode. The extent of mineralisation of the 4-nitrophenol was evaluated on a TOC analyser after a period of $150 \mathrm{~min}$. The TOC results showed a removal percentage of $58 \%$ for Pd-ZnO-EG electrode which is better in comparison with that of EG (32\%) and $\mathrm{ZnO}$ EG (40\%) electrodes.

Optimisation of the reaction conditions was carried out by evaluating the influence of change in $\mathrm{pH}$, and current density. The photoelectrocatalytic removal of the 4-nitrophenol using Pd-ZnO-EG was found to be $\mathrm{pH}$ dependent as presented in the kinetics curves in Fig. 4c. The results obtained at $\mathrm{pH} \mathrm{4,} 7$ and 10 are 60, 94 and 64\%, respectively. With a pKa of 7.2, 4-nitrophenol is expected to be neutral at $\mathrm{pH}$ 7. The highest degradation rate obtained from $\mathrm{pH} 7$ may be as a result of the prevailing neutral 4-nitrophenol which is not poised to or less likely to compete with the $\mathrm{OH}$ radical. The influence of current density, which is generally proportional to the production of hydroxyl radical, ${ }^{63}$ on the removal efficiency of 4-nitrophenol on the PdZnO-EG electrode was investigated (Fig. 4d). As the current density was increased from 2.5 to $5 \mathrm{~mA} \mathrm{~cm}{ }^{-2}$, the removal efficiency increased from 73 to $94 \%$. However, no significant increase in the removal efficiency was observed when the current density was increased to $7.5 \mathrm{~mA} \mathrm{~cm}{ }^{-2}$. This could be attributed to increase in oxygen evolution which tends to compete with the oxidation of the organic pollutant. ${ }^{63}$ The production of intermediates during the degradation process can constitute a film on the surface of the electrode and reduce its activity. Consequently, increasing the current density further from 5 to $7.5 \mathrm{~mA} \mathrm{~cm}{ }^{-2}$ would not lead to effectiveness of cost and energy for the photoelectrocatalytic removal of the 4nitrophenol since there is no large difference in the increase in the removal of 4-nitrophenol at a current density of $7.5 \mathrm{~mA}$ $\mathrm{cm}^{-2}$. Thus, a current density of $5 \mathrm{~mA} \mathrm{~cm}{ }^{-2}$ was employed for the experiments for energy efficiency.

\subsection{Photoelectrocatalytic degradation kinetics and mechanism}

The rate of reaction of the degradation of 4-nitrophenol at the EG, ZnO-EG and Pd-ZnO-EG electrode were investigated by modelling the obtained data with Langmuir-Hinshelwood firstorder kinetics equation;

$$
r=\mathrm{d} C / \mathrm{d} t=k_{\mathrm{r}} K_{\mathrm{ad}} C /\left(1+K_{\mathrm{ad}} C\right)
$$

where $r$ is the degradation rate, $C$ is the concentration of 4nitrophenol at a particular time, $t, K_{\mathrm{ad}}$ is the reactant coefficient of adsorption, and $k_{\mathrm{r}}$ is the rate constant of reaction. At very low initial concentration and relatively weak adsorption, $K_{\mathrm{ad}} C$ is $\ll$ 1 , and the equation can be simplified to apparent first-order kinetic having $k_{\text {app }}$ as the apparent first-order rate constant;

$$
\ln C_{0} / C_{t}=k_{\mathrm{r}} K_{\mathrm{ad}} t=k_{\mathrm{app}} t
$$

$C_{0}$ is the 4-nitrophenol initial concentration, $C_{t}$ is the its concentration at reaction time, $t$. Fig. 5a depicts that the $\ln C_{0} /$ $C_{t}$ versus time curves of the obtained data fitted to give linear kinetics curves with coefficients of correlation greater than 0.95. This implies that reaction for the photoelectrocatalytic removal of 4-nitrophenol follows the pseudo first-order reaction kinetics model, and it can be seen that the Pd-ZnO-EG electrode displayed a better rate of degradation in comparison to EG and ZnO-EG electrodes. The estimated rate constants and correlation coefficients for the electrodes are $5.53 \times 10^{-3} \mathrm{~min}^{-1}$ and 0.9753 for EG; $6.97 \times 10^{-3} \mathrm{~min}^{-1}$ and 0.9928 for ZnO-EG; and $18.52 \times 10^{-3} \mathrm{~min}^{-1}$ and 0.9913 for Pd-ZnO-EG.

A plausible mechanism was proposed to describe the photoelectrocatalytic process and the reactions taking place for the 4-nitrophenol removal are presented in eqn (4)-(9);

$$
\begin{gathered}
h v+\mathrm{ZnO} \rightarrow \mathrm{ZnO}\left(\mathrm{h}_{\mathrm{VB}}{ }^{+}+\mathrm{e}_{\mathrm{CB}}{ }^{-}\right) \\
\mathrm{e}^{-}+\mathrm{O}_{2} \rightarrow \mathrm{O}_{2}^{-} \\
\mathrm{h}^{+}+\mathrm{H}_{2} \mathrm{O} \rightarrow \mathrm{H}^{+}+{ }^{\cdot} \mathrm{OH}
\end{gathered}
$$

(a)

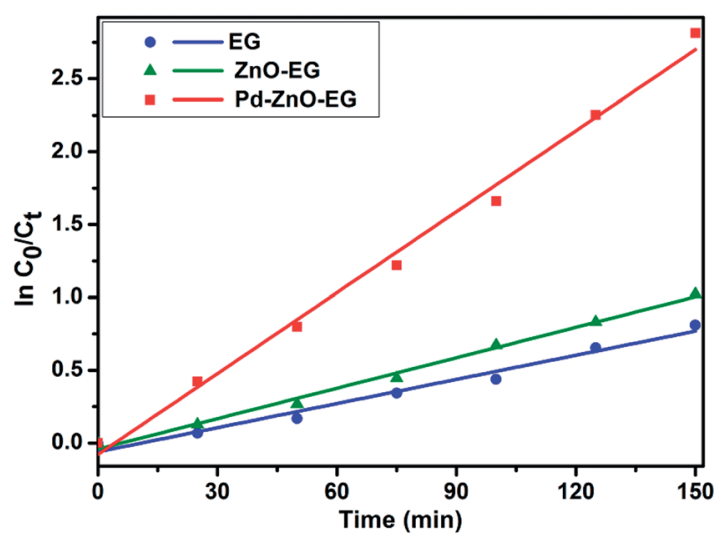

(b)

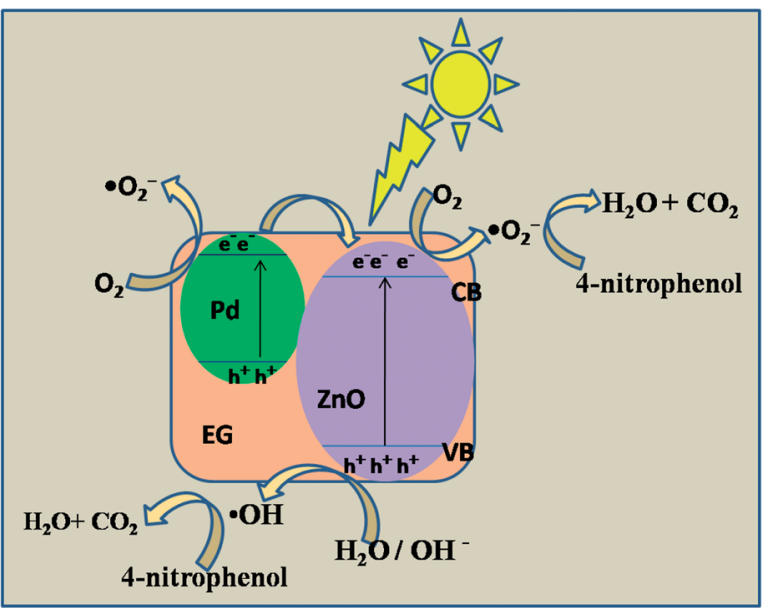

Fig. 5 (a) Removal kinetics plots of EG, ZnO-EG and Pd-ZnO-EG for the photo-electrocatalytic removal of 4-nitrophenol at neutral $\mathrm{pH}$ and $7 \mathrm{~mA} \mathrm{~cm}^{-2}$ at Pd-ZnO-EG electrode. (b) Proposed mechanism for the charge transfer involved in the photo-electrocatalytic activity of $\mathrm{Pd}-$ $\mathrm{ZnO}-\mathrm{EG}$ electrode for the removal of 4-nitrophenol. 


$$
\begin{gathered}
\cdot \mathrm{O}_{2}{ }^{-}+2 \mathrm{H}^{+} \rightarrow \cdot \mathrm{OH}+{ }^{\cdot} \mathrm{OH} \\
\cdot \mathrm{OH}+\text { 4-nitrophenol } \rightarrow \text { intermediates } \rightarrow \mathrm{CO}_{2}+\mathrm{H}_{2} \mathrm{O} \\
\cdot \mathrm{O}_{2}{ }^{-}+\text {4-nitrophenol } \rightarrow \text { intermediates } \rightarrow \mathrm{CO}_{2}+\mathrm{H}_{2} \mathrm{O}
\end{gathered}
$$

The difference in the photoelectrocatalytic performance of the different electrodes can be explained using the difference in the work function of $\mathrm{ZnO}(5.2 \mathrm{eV})$ and $\mathrm{Pd}(5.12 \mathrm{eV})$. It is generally known that a Schottky junction is formed when two materials having different work functions are connected. Electrons flow from the low work function material to the one with a higher work function. ${ }^{\mathbf{6 4}, \mathbf{6 5}}$ Since the Fermi energy of Pd metal (lower work function) is higher than the Fermi level of $\mathrm{ZnO}$ semiconductor (higher work function), electrons tend to transfer from the Pd to the ZnO (Fig. 5b) until a new Fermi energy level is achieved at equilibrium. At this new Fermi energy level, an Ohmic-type junction which is a metal-semiconductor Schottky barrier is formed under equilibrium. ${ }^{\mathbf{2 4 , 6 4 , 6 5}}$ Upon irradiation with visible light, electrons and holes are photoexcited in the $\mathrm{ZnO}$ and the photoinduced electrons in the conduction band of the $\mathrm{ZnO}$ are transferred to the Pd under the influence of the static electric field at the Schottky junction, ${ }^{24,65}$ thus, an effective charge separation is facilitated. The separated and the remaining electrons on the $\mathrm{ZnO}$ conduction band react with dissolved oxygen molecules to generate superoxide radicals which react with water molecules to give hydroxyl radicals. The photoinduced holes can migrate to the surface of the $\mathrm{ZnO}$ and react with water on the semiconductor surface to form hydroxyl radicals. Based on eqn (7)-(9), these generated radicals further react with the 4-nitrophenol pollutant to give intermediates which undergo mineralisation to produce $\mathrm{CO}_{2}$ and $\mathrm{H}_{2} \mathrm{O}$.

\subsection{Computational modelling of the degradation of 4- nitrophenol}

The degradation reaction mechanisms of the oxidation of 4nitrophenol were studied using the B3LYP/6-31G(d) method in the gas phase based on the reaction between 4-nitrophenol and hydroxyl radical. ${ }^{38,39}$ The reaction pathways for the attack at different positions of 4-nitrophenol by hydroxyl radical were considered and these are represented in Fig. 6a. The optimized geometries of the transition states are illustrated in Fig. 6b. The intrinsic reaction coordinates are provided as ESI in Fig. $\mathrm{S} 2 \dagger$ is found from previous reports that molecular oxygen which is evolved at the cathode in an electrochemical system plays an important role in the degradation process and therefore, subsequent reactions with molecular oxygen were also investigated. ${ }^{\mathbf{6 6 , 6 7}}$

It was found that the reaction of hydroxyl radical with the 4nitrophenol has low activation energy and pathway $\mathrm{D}$ is

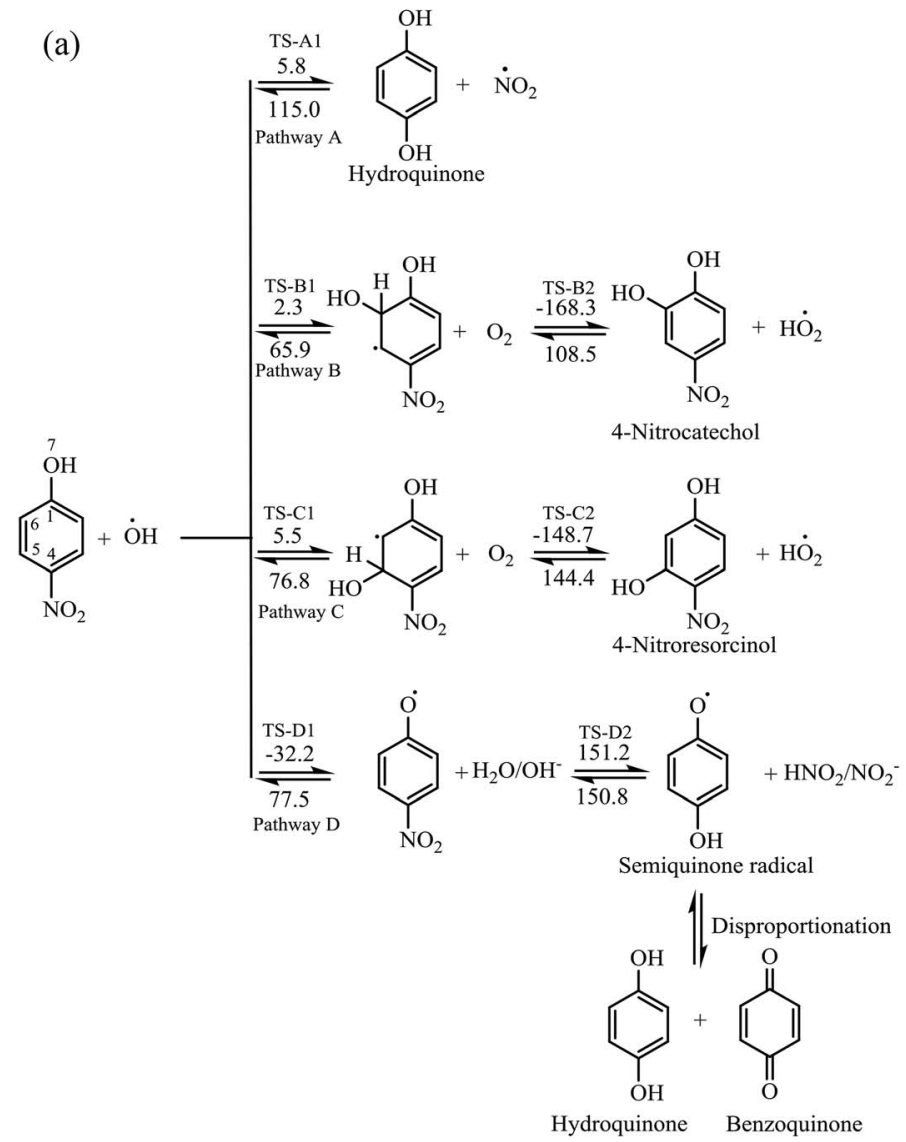

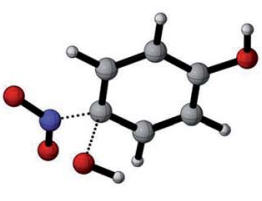

TS-Al

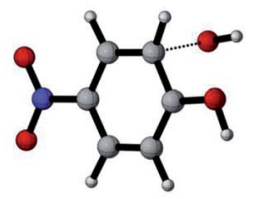

TS-B1

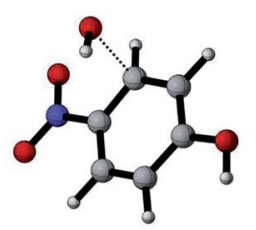

TS-C1

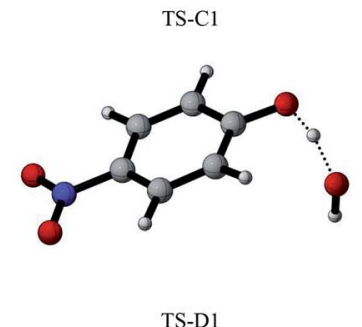

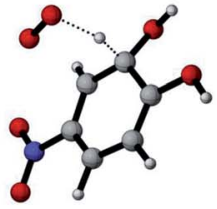

TS-B2

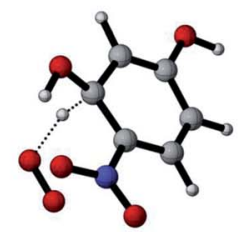

TS-C2

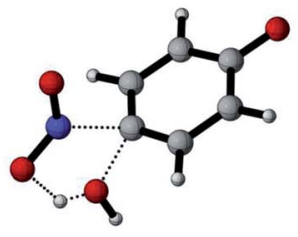

TS-D2

Fig. 6 (a) Oxidative degradation reaction pathways for the hydroxyl attack and hydrogen abstraction on 4-nitrophenol to give 4-nitrocatechol, 4-nitroresorcinol and semiquinone radical which eventually disproportionate into hydroquinone and benzoquinone (activation energies (numbers on arrows) for the forward and backward reactions are given in $\mathrm{kJ} \mathrm{mol}^{-1}$ ). (b) Optimized geometries of the transition states. 
barrierless. The addition of hydroxyl radical at position C-4 leads to the formation of hydroquinone and $\mathrm{NO}_{2}$ radical, having activation energy of $5.8 \mathrm{~kJ} \mathrm{~mol}^{-1}$ for the forward reaction and $115.0 \mathrm{~kJ} \mathrm{~mol}^{-1}$ for the reverse reaction (Fig. 6a).

The pathways (B and C) of the hydroxyl radical attack on the ortho and meta positions of 4-nitrophenol have low activation energies of 2.3 and $5.5 \mathrm{~kJ} \mathrm{~mol}^{-1}$, respectively. Subsequently, the

(a)

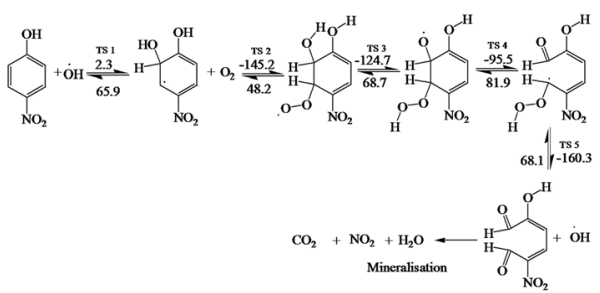

(b)

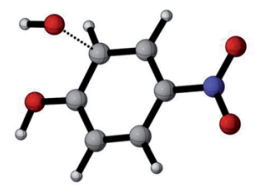

TS1

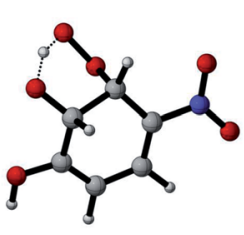

TS3

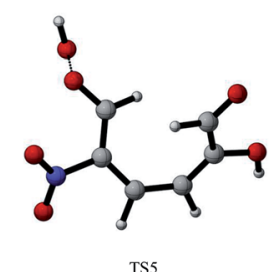

(c)

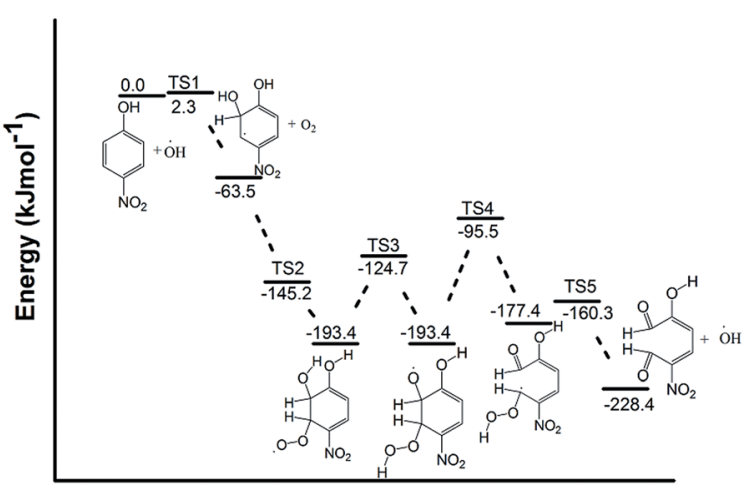

Fig. 7 (a) Plausible mechanism for the 4-nitrophenol oxidative degradation reaction leading to the ring opening of the molecule (activation energies (numbers on arrows) for the forward and backward reactions are given in $\mathrm{kJ} \mathrm{mol}^{-1}$, the activation energies of TS2TS5 were calculated with respect to the 4-nitrocatechol radical and $\mathrm{O}_{2}$ as reactants). (b) Transition states at various steps of the ring opening of 4-nitrophenol. (c) Energy profile for the ring opening reactions. substitution reaction was accompanied by proton abstraction by molecular oxygen produced in the electrochemical system/ solution. This leads to the production of 4-nitrocatechol, 4nitroresorcinol and hydroperoxyl radical $\left(\mathrm{HO}_{2}{ }^{\circ}\right)$. 4-Nitrocatechol and 4-nitroresorcinol are some of the oxidation products of 4-nitrophenol reported in literature, where 4nitrocatechol is preferentially formed as this pathway is kinetically favoured. ${ }^{68-71}$ These results are also in agreement with the products obtained for the computational studies for the degradation of 4-chlorophenol leading to 4-chlorocatechol and hydroperoxyl radical. ${ }^{66}$ In addition, the molecular oxygen can also react with the ortho $\mathrm{OH}$ adduct to form peroxyl radicals and eventually, leads to the ring opening and release hydroperoxyl radical (Fig. 7). The ring opening gives rise to intermediates that are oxidised by $\mathrm{OH}$ radical and mineralised into carbon dioxide, water and nitrates. ${ }^{6,70}$

Another possible degradation pathway is the abstraction of proton from the hydroxyl group on 4-nitrophenol (pathway D). This pathway is found barrierless and can occur spontaneously, leading to the production of 4-nitrophenoxyl radical and water. The nitrophenoxyl radical and water further reacts to generate a semiquinone radical which was found to be endothermic and slow due to the large activation energy computed $\left(151.2 \mathrm{~kJ} \mathrm{~mol}^{-1}\right)$. Semiquinone radicals are generally known to break down into hydroquinone and 1,4-benzoquinone which are other oxidation products of 4-nitrophenol which were determined in several experimental studies. ${ }^{\mathbf{6 6 , 6 8 - 7 0 , 7 2}}$ The energy profile for the pathway A is found in the supplementary information Fig. S3. $\dagger$

It can be deduced from the DFT study that for the photoelectrocatalytic degradation of 4-nitrophenol by hydroxyl radical generated in the system led to the formation of benzoquinone, hydroquinone, 4-nitrocatechol and 4-nitroresorcinol. Reaction with the ortho $\mathrm{OH}$ adduct can also cause ring opening which would be further oxidised or mineralised to less harmful products. In addition, there is generation of hydroperoxyl radical and hydroxyl radical in the system which can initiate more attacks.

\section{Conclusion}

In summary, Pd-ZnO-EG electrode was constructed from a PdZnO-EG nanocomposite, synthesised by a hydrothermal method and characterised using various techniques. The PdZnO-EG nanomaterials have large surface area, pore size and volume, and strong absorption in the visible light region. The electrode was used for the photoelectrocatalytic removal of 4nitrophenol as a target water pollutant. The Pd-ZnO-EG electrode showed improved photoelectrocatalytic activity in relation to ZnO-EG and EG electrodes for the removal of the 4-nitrophenol. This is as a result of the Pd nanoparticles and the conducting EG acting as collectors for the photoexcited electrons, thereby aiding charge transfer and reducing recombination of charges. In addition, the photocurrent responses showed that the Pd-ZnO-EG nanocomposite electrode could be employed as a good photo-electrode for the benefits of photoelectrocatalytic processes and environmental remediation such 
as treatment of industrial wastewaters. The oxidative degradation of 4-nitrophenol by hydroxyl radical was predicted using DFT method which led to the generation of hydroquinone, benzoquinone, 4-nitrocatechol, 4-nitroresorcinol and the ring opening of the 4-nitrophenol. This was obtained by DFT computations of plausible reaction mechanism and pathways resulting from hydroxyl radical attacks on different positions on the molecule of 4-nitrophenol, accompanied by hydrogen abstraction by ground state oxygen reactions. Furthermore, hydroxyl radical was regenerated which can further oxidised the ring structure and initiates a new degradation process.

\section{Conflicts of interest}

There are no conflicts of interest to declare.

\section{Acknowledgements}

The authors wish to acknowledge financial supports from the following institutions: The Faculty of Science and Centre for Nanomaterials Science Research, University of Johannesburg, South Africa; Obafemi Awolowo University, Ile-Ife, Nigeria National Research Foundation of South Africa (CPRR Grant number: 98887); Water Research Commission of South Africa (Grant Number: K5/2567) and the L'Oreal-UNESCO For Women in Science. The authors wish to thank Prof Jaromír Jirkovský from the Institute of Physical Chemistry, v.i.i., Academy of Sciences of the Czech Republic, Dolejskova 3, 18223 Prague 8, Czech Republic, for his guidance and technical assistance. The Computational Chemistry Laboratory, University of Mauritius is gratefully acknowledged for the computational software used. The postgraduate students in that laboratory are acknowledged for their assistance.

\section{References}

$1 \mathrm{Z}$. Wu, Y. Zhang, X. Wang and Z. Zou, Ag@ $\mathrm{SrTiO}_{3}$ nanocomposite for super photocatalytic degradation of organic dye and catalytic reduction of 4-nitrophenol, New J. Chem., 2017, 41(13), 5678-5687.

2 J. Ding, Q. Li, L. Zhao, X. Li, Q. Yue and B. Gao, A wheat straw cellulose based semi-IPN hydrogel reactor for metal nanoparticles preparation and catalytic reduction of 4nitrophenol, RSC Adv., 2017, 7(29), 17599-17611.

3 N. Sahiner, H. Ozay, O. Ozay and N. Aktas, New catalytic route: hydrogels as templates and reactors for in situ $\mathrm{Ni}$ nanoparticle synthesis and usage in the reduction of 2-and 4-nitrophenols, Appl. Catal., A, 2010, 385(1), 201-207.

4 R. Zhu, Q. Chen, Q. Zhou, Y. Xi, J. Zhu and H. He, Adsorbents based on montmorillonite for contaminant removal from water: a review, Appl. Clay Sci., 2016, 123, 239-258.

5 S. Ammar, N. Oturan and M. A. Oturan, Electrochemical oxidation of 2-nitrophenol in aqueous medium by electroFenton technology, J. Environ. Eng. Manage., 2007, 17(2), 89. 6 P. Gharbani, S. Tabatabaii and A. Mehrizad, Removal of Congo red from textile wastewater by ozonation, Int. J. Environ. Sci. Technol., 2008, 5(4), 495-500.
7 L.-Z. Huang, H. C. B. Hansen and M. J. Bjerrum, Electrochemical reduction of nitroaromatic compounds by single sheet iron oxide coated electrodes, J. Hazard. Mater., 2016, 306, 175-183.

8 A. K. Verma, R. R. Dash and P. Bhunia, A review on chemical coagulation/flocculation technologies for removal of colour from textile wastewaters, J. Environ. Manage., 2012, 93(1), 154-168.

9 E. Brillas and C. A. Martínez-Huitle, Decontamination of wastewaters containing synthetic organic dyes by electrochemical methods. An updated review, Appl. Catal., $B, 2015,166,603-643$.

10 G. R. Malpass, D. W. Miwa, S. A. Machado and A. J. Motheo, SnO 2-based materials for pesticide degradation, J. Hazard. Mater., 2010, 180(1), 145-151.

11 M. Peleyeju, E. Umukoro, J. Babalola and O. Arotiba, Electrochemical Degradation of an Anthraquinonic Dye on an Expanded Graphite-Diamond Composite Electrode, Electrocatalysis, 2016, 7(2), 132-139.

12 K. Jüttner, U. Galla and H. Schmieder, Electrochemical approaches to environmental problems in the process industry, Electrochim. Acta, 2000, 45(15), 2575-2594.

13 E. Brillas and C. A. Martínez-Huitle, Decontamination of wastewaters containing synthetic organic dyes by electrochemical methods. An updated review, Appl. Catal., $B$ 2015, 166-167, 603-643, DOI: 10.1016/ j.apcatb.2014.11.016.

14 E. Kusmierek and E. Chrzescijanska, Application of TiO 2RuO 2/Ti electrodes modified with WO 3 in electro-and photoelectrochemical oxidation of Acid Orange 7 dye, $J$. Photochem. Photobiol., A, 2015, 302, 59-68.

15 E. H. Umukoro, M. G. Peleyeju, J. C. Ngila and O. A. Arotiba, Towards wastewater treatment: Photo-assisted electrochemical degradation of 2-nitrophenol and orange II dye at a tungsten trioxide-exfoliated graphite composite electrode, Chem. Eng. J., 2017, 317, 290-301.

16 H. W. Jeong, T. H. Jeon, J. S. Jang, W. Choi and H. Park, Strategic modification of $\mathrm{BiVO}_{4}$ for improving photoelectrochemical water oxidation performance, J. Phys. Chem. C, 2013, 117(18), 9104-9112.

17 P. Y. Kuang, J. R. Ran, Z. Q. Liu, H. J. Wang, N. Li, Y. Z. Su, Y. G. Jin and S. Z. Qiao, Enhanced photoelectrocatalytic activity of BiOI nanoplate-zinc oxide nanorod $\mathrm{p}-\mathrm{n}$ heterojunction, Chem.-Eur. J., 2015, 21(43), 15360-15368.

$18 \mathrm{~J} . \mathrm{Li}, \mathrm{S} . \mathrm{Lv}, \mathrm{Y}$. Liu, J. Bai, B. Zhou and $\mathrm{X} . \mathrm{Hu}$, Photoeletrocatalytic activity of an n-ZnO/p-Cu 2 O/n-TNA ternary heterojunction electrode for tetracycline degradation, J. Hazard. Mater., 2013, 262, 482-488.

19 E. H. Umukoro, M. G. Peleyeju, J. C. Ngila and O. A. Arotiba, Photoelectrochemical degradation of orange II dye in wastewater at a silver-zinc oxide/reduced graphene oxide nanocomposite photoanode, RSC Adv., 2016, 6(58), 5286852877.

20 A. Zhu, Q. Zhao, X. Li and Y. Shi, $\mathrm{BiFeO}_{3} / \mathrm{TiO}_{2}$ nanotube arrays composite electrode: Construction, characterization, and enhanced photoelectrochemical properties, ACS Appl. Mater. Interfaces, 2013, 6(1), 671-679. 
21 J. Becker, K. R. Raghupathi, J. St. Pierre, D. Zhao and R. T. Koodali, Tuning of the crystallite and particle sizes of $\mathrm{ZnO}$ nanocrystalline materials in solvothermal synthesis and their photocatalytic activity for dye degradation, $J$. Phys. Chem. C, 2011, 115(28), 13844-13850.

22 X. Chen, Y. He, Q. Zhang, L. Li, D. Hu and T. Yin, Fabrication of sandwich-structured $\mathrm{ZnO} /$ reduced graphite oxide composite and its photocatalytic properties, J. Mater. Sci., 2010, 45(4), 953-960.

23 F. Sun, X. Qiao, F. Tan, W. Wang and X. Qiu, One-step microwave synthesis of $\mathrm{Ag} / \mathrm{ZnO}$ nanocomposites with enhanced photocatalytic performance, J. Mater. Sci., 2012, $1-7$.

24 L. Zhang, L. Du, X. Yu, S. Tan, X. Cai, P. Yang, Y. Gu and W. Mai, Significantly enhanced photocatalytic activities and charge separation mechanism of Pd-decorated ZnOgraphene oxide Nanocomposites, ACS Appl. Mater. Interfaces, 2014, 6(5), 3623-3629.

25 S. Balachandran, N. Prakash and M. Swaminathan, Heteroarchitectured $\mathrm{Ag}-\mathrm{Bi}_{2} \mathrm{O}_{3}-\mathrm{ZnO}$ as a bifunctional nanomaterial, $R S C A d v .$, 2016, 6(24), 20247-20257.

26 E. H. Umukoro, M. G. Peleyeju, J. C. Ngila and O. A. Arotiba, Photocatalytic degradation of acid blue 74 in water using Ag$\mathrm{Ag}_{2} \mathrm{O}-\mathrm{Zno}$ nanostuctures anchored on graphene oxide, Solid State Sci., 2016, 51, 66-73.

27 W. W. Wang, Y. J. Zhu and L. X. Yang, $\mathrm{ZnO}-\mathrm{SnO}_{2}$ hollow spheres and hierarchical nanosheets: hydrothermal preparation, formation mechanism, and photocatalytic properties, Adv. Funct. Mater., 2007, 17(1), 59-64.

28 H. Gu, Y. Yang, J. Tian and G. Shi, Photochemical synthesis of noble metal (Ag, Pd, Au, Pt) on graphene/ZnO multihybrid nanoarchitectures as electrocatalysis for $\mathrm{H}_{2} \mathrm{O}_{2}$ reduction, ACS Appl. Mater. Interfaces, 2013, 5(14), 6762-6768.

29 S. Ma, J. Xue, Y. Zhou and Z. Zhang, Photochemical synthesis of $\mathrm{ZnO} / \mathrm{Ag}_{2} \mathrm{O}$ heterostructures with enhanced ultraviolet and visible photocatalytic activity, J. Mater. Chem. A, 2014, 2(20), 7272-7280.

30 Y. Wang, Q. Wang, X. Zhan, F. Wang, M. Safdar and J. He, Visible light driven type II heterostructures and their enhanced photocatalysis properties: a review, Nanoscale, 2013, 5(18), 8326-8339.

31 W. Shi, Y. Yan and X. Yan, Microwave-assisted synthesis of nano-scale $\mathrm{BiVO}_{4}$ photocatalysts and their excellent visiblelight-driven photocatalytic activity for the degradation of ciprofloxacin, Chem. Eng. J., 2013, 215, 740-746.

$32 \mathrm{M}$. Yi and Z. Shen, Kitchen blender for producing highquality few-layer graphene, Carbon, 2014, 78, 622-626.

$33 \mathrm{M}$. Yi and Z. Shen, A review on mechanical exfoliation for the scalable production of graphene, J. Mater. Chem. A, 2015, 3(22), 11700-11715.

$34 \mathrm{M}$. Yi, Z. Shen and J. Zhu, A fluid dynamics route for producing graphene and its analogues, Chin. Sci. Bull., 2014, 59(16), 1794-1799.

35 T. Ndlovu, B. B. Mamba, S. Sampath, R. W. Krause and O. A. Arotiba, Voltammetric detection of arsenic on a bismuth modified exfoliated graphite electrode, Electrochim. Acta, 2014, 128, 48-53.
36 B. Ntsendwana, B. B. Mamba, S. Sampath and O. A. Arotiba, Synthesis, characterisation and application of an exfoliated graphite-diamond composite electrode in the electrochemical degradation of trichloroethylene, RSC Adv., 2013, 3(46), 24473-24483.

37 M. G. Peleyeju, A. O. Idris, E. H. Umukoro, J. O. Babalola and O. A. Arotiba, Electrochemical Detection of 2, 4Dichlorophenol on a Ternary Composite Electrode of Diamond, Graphene, and Polyaniline, ChemElectroChem, 2017, 4, 1074-1080.

38 A. D. Becke, Density-functional thermochemistry. I. The effect of the exchange-only gradient correction, J. Chem. Phys., 1992, 96(3), 2155-2160.

39 C. Lee, W. Yang and R. Parr, Density-functional exchangeenergy approximation with correct asymptotic behaviour, Phys. Rev. B, 1988, 37, 785-789.

$40 \mathrm{~W}$. J. Hehre, $A b$ initio molecular orbital theory, WileyInterscience, 1986.

41 C. Gonzalez and H. Schelgel, Reaction path following in mass-weighted internal coordinates, J. Phys. Chem., 1990, 94(14), 5523-5527.

42 C. Gonzalez and H. B. Schlegel, An improved algorithm for reaction path following, J. Chem. Phys., 1989, 90(4), 21542161.

43 M. Frisch; G. Trucks; H. Schlegel; G. Scuseria; M. Robb; J. Cheeseman; G. Scalmani; V. Barone; B. Mennucci; G. Petersson. Gaussian 09, Revision E. 01, Gaussian. Inc., Wallingford CT, 2009.

44 R. Dooley, G. Allen and S. Pamidighantam, in Computational chemistry grid: production cyberinfrastructure for computational chemistry, Proceedings of the 13th Annual Mardi Gras Conference, 2005, p. 83.

45 R. Dooley, K. Milfeld, C. Guiang, S. Pamidighantam and G. Allen, From proposal to production: Lessons learned developing the computational chemistry grid cyberinfrastructure, J. Grid Comput., 2006, 4(2), 195-208.

46 K. Milfeld, C. Guiang, S. Pamidighantam and J. Giuliani, in Cluster computing through an application-oriented computational chemistry Grid, Proceedings of the 2005 Linux Clusters: The HPC Revolution, 2005.

47 A. Khan, Raman spectroscopic study of the ZnO nanostructures, Journal of Pakistan Materials Society, 2010, 4, 5-9.

48 A. Khan and M. E. Kordesch, Synthesis of novel zinc oxide microphone-like microstructures, Mater. Lett., 2008, 62(2), 230-234.

49 A. Umar, B. Karunagaran, E. Suh and Y. Hahn, Structural and optical properties of single-crystalline $\mathrm{ZnO}$ nanorods grown on silicon by thermal evaporation, Nanotechnology, 2006, 17(16), 4072.

50 A.-S. Mamede, G. Leclercq, E. Payen, J. Grimblot and P. Granger, Surface Raman spectroscopic study of NO transformation over Pd-based catalysts, Phys. Chem. Chem. Phys., 2003, 5(20), 4402-4406.

51 X. Yang, M. Zhen, G. Li, X. Liu, X. Wang, C. Shu, L. Jiang and C. Wang, Preparation of Pd-decorated fullerenes on carbon 
nanotubes with excellent electrocatalytic properties in alkaline media, J. Mater. Chem. A, 2013, 1(28), 8105-8110.

52 K.-W. Park and J. H. Jung, Spectroscopic and electrochemical characteristics of a carboxylated graphene- $\mathrm{ZnO}$ composites, J. Power Sources, 2012, 199, 379-385.

53 P. Wang, Y. Tang, Z. Dong, Z. Chen and T.-T. Lim, Ag-AgBr/ TiO 2/RGO nanocomposite for visible-light photocatalytic degradation of penicillin G, J. Mater. Chem. A, 2013, 1(15), 4718-4727.

54 P. Avetta, M. Sangermano, M. Lopez-Manchado and P. Calza, Use of graphite oxide and/or thermally reduced graphite oxide for the removal of dyes from water, J. Photochem. Photobiol., A, 2015, 312, 88-95.

55 L. Hao, W. Gao, Y. Liu, Y. Liu, Z. Han, Q. Xue and J. Zhu, Selfpowered broadband, high-detectivity and ultrafast photodetectors based on Pd-MoS 2/Si heterojunctions, Phys. Chem. Chem. Phys., 2016, 18(2), 1131-1139.

56 P. Zuppella, E. Pasqualotto, S. Zuccon, F. Gerlin, A. J. Corso, M. Scaramuzza, A. De Toni, A. Paccagnella and M. G. Pelizzo, Palladium on plastic substrates for plasmonic devices, Sensors, 2015, 15(1), 1138-1147.

57 Y. S. Grewal, M. J. Shiddiky, S. A. Gray, K. M. Weigel, G. A. Cangelosi and M. Trau, Label-free electrochemical detection of an Entamoeba histolytica antigen using cellfree yeast-scFv probes, Chem. Commun., 2013, 49(15), 15511553.

58 W. Yuan, Y. Zhou, Y. Li, C. Li, H. Peng, J. Zhang, Z. Liu, L. Dai and G. Shi, The edge-and basal-plane-specific electrochemistry of a single-layer graphene sheet, Sci. Rep., 2013, 3, 2248.

59 F. Wang, W. Li, S. Gu, H. Li, X. Wu and X. Liu, Samarium and Nitrogen Co-Doped $\mathrm{Bi}_{2} \mathrm{WO}_{6}$ Photocatalysts: Synergistic Effect of Sm3+/Sm2+ Redox Centers and N-Doped Level for Enhancing Visible-Light Photocatalytic Activity, Chem.-Eur. J., 2016, 22(36), 12859-12867.

60 W. Zhao, Y. Liu, Z. Wei, S. Yang, H. He and C. Sun, Fabrication of a novel $\mathrm{p}-\mathrm{n}$ heterojunction photocatalyst nBivO 4@ p- $\mathrm{MoS}_{2}$ with core-shell structure and its excellent visible-light photocatalytic reduction and oxidation activities, Appl. Catal., B, 2016, 185, 242-252.

61 M. Fan, C. Yang, W. Pu and J. Zhang, Liquid phase deposition of $\mathrm{ZnO}$ film for photoelectrocatalytic degradation of p-nitrophenol, Mater. Sci. Semicond. Process., 2014, 17, 104-109.
62 J. bo Zhong, J. zhang Li, X. yang He, J. Zeng, Y. Lu, W. Hu and K. Lin, Improved photocatalytic performance of Pd-doped ZnO, Curr. Appl. Phys., 2012, 12(3), 998-1001.

63 Y. Wang, C. Shen, M. Zhang, B.-T. Zhang and Y.-G. Yu, The electrochemical degradation of ciprofloxacin using a SnO 2-Sb/Ti anode: Influencing factors, reaction pathways and energy demand, Chem. Eng. J., 2016, 296, 79-89.

64 S. A. Ansari, M. M. Khan, M. O. Ansari, J. Lee and M. H. Cho, Biogenic synthesis, photocatalytic, and photoelectrochemical performance of $\mathrm{Ag}-\mathrm{ZnO}$ nanocomposite, J. Phys. Chem. C, 2013, 117(51), 2702327030.

65 D. R. Hang, S. E. Islam, C. H. Chen and K. H. Sharma, Full Solution-Processed Synthesis and Mechanisms of a Recyclable and Bifunctional Au/ZnO Plasmonic Platform for Enhanced UV/Vis Photocatalysis and Optical Properties, Chem.-Eur. J., 2016, 22(42), 14950-14961.

66 V.-M. Guérin, R. Zouzelka, H. Bibova-Lipsova, J. Jirkovsky, J. Rathousky and T. Pauporté, Experimental and DFT study of the degradation of 4-chlorophenol on hierarchical micro-/nanostructured oxide films, Appl. Catal., B, 2015, 168, 132-140.

67 Y.-y. Zhao, E. Tsuchida, Y.-K. Choe, J. Wang, T. Ikeshoji and A. Ohira, Theoretical studies on the degradation of hydrocarbon copolymer ionomers used in fuel cells, $J$. Membr. Sci., 2015, 487, 229-239.

68 S. Chaliha, K. G. Bhattacharyya and P. Paul, Oxidation of 4nitrophenol in water over Fe (III), Co (II), and Ni (II) impregnated MCM41 catalysts, J. Chem. Technol. Biotechnol., 2008, 83(10), 1353-1363.

69 A. Chauhan, G. Pandey, N. K. Sharma, D. Paul, J. Pandey and R. K. Jain, p-Nitrophenol degradation via 4-nitrocatechol in Burkholderia sp. SJ98 and cloning of some of the lower pathway genes, Environ. Sci. Technol., 2010, 44(9), 34353441.

70 R. Ding, Z.-Y. Mao and J.-L. Wang, Synergistic effects of 4nitrophenol degradation using gamma irradiation combined with a advanced oxidation process, Nucl. Sci. Tech., 2016, 27(1), 4.

71 V. Kadiyala, B. F. Smets, K. Chandran and J. C. Spain, High affinity p-nitrophenol oxidation by Bacillus sphaericus JS905, FEMS Microbiol. Lett., 1998, 166(1), 115-120.

$72 \mathrm{~J}$. Li, Q. Liu, Q. qing Ji and B. Lai, Degradation of pnitrophenol (PNP) in aqueous solution by $\mathrm{Fe} 0$-PM-PS system through response surface methodology (RSM), Appl. Catal., B, 2017, 200, 633-646. 